\title{
Análisis del espacio público versus privado en docentes no universitarios españoles
}

\section{Analysis of the public versus private space in Spanish teachers}

\author{
Yolanda Rodríguez Castro ${ }^{1}$, María Lameiras Fernández y María Victoria Carrera Fernández \\ Universidad de Vigo, España
}

(Recepción: Noviembre 2008 - Aceptación: Abril 2009)

\begin{abstract}
Resumen
El objetivo de este estudio es analizar la división del espacio público y privado del colectivo docente no universitario español, teniendo en cuenta la tipología del centro educativo. La muestra representativa a nivel nacional, está compuesta por 744 docentes (62.5\% son profesoras y 37.4\% son profesores), con una media de edad de 41.3 (SD: 9.9). Se aplicaron los siguientes cuestionarios: de variables sociodemográficas y de Tiempo dedicado a las tareas domésticas (adaptado de Martínez, 1995). Se confirma la presencia masiva de la mujer en el ámbito educativo, sobretodo en la Educación Primaria, aunque son los profesores los que ostentan los cargos de mayor responsabilidad, independientemente de la tipología del centro educativo. Y que siguen siendo las profesoras las que sobrellevan una mayor carga en el desarrollo de las tareas domésticas. Esto evidencia la clara asimetría entre los géneros en la división del espacio público versus privado en el colectivo docente.
\end{abstract}

Palabras clave: espacio público, espacio privado, tareas domésticas, docentes.

\begin{abstract}
Abstrat
The aim of this study is to analyze the division of public and private space in a group of 744 Spanish teachers (62.5\% are female and $37.4 \%$ are male) with an average age of 41.3 years old (SD: 9.9), and responding to a questionnaire package: the socio-demographic variables and Time spent on housework (adapted from Martinez, 1995). The results showed that there were very much female teachers in Education, especially in Primary Education. But male teachers play the highest positions of responsibility in their work. Most female teachers spend more time on household chores that male teachers. This shows that there are differences between genders in the division of public and private space within the collective teacher.
\end{abstract}

Key-words: public and private space, housework, teachers.

1 Correspondencia a: Yolanda Rodríguez Castro, Universidad de Vigo, Facultad de Ciencias de la Educación, Pabellón 2. Campus de Ourense, Av. Castelao sn, 32004 Ourense (España). E-mail: yrcastro@uvigo.es 


\section{Introducción}

Con la revolución industrial se propicia una nueva realidad económica que deja atrás las sociedades ganaderas y agrícolas para imponer la "venta” de la fuerza de trabajo como la principal fuente de ingresos y de subsistencia para la población. Lo que propicia la segregación sexuada de tiempos y espacios de producción (empresa, fábrica) y reproducción (familia). Esta nueva realidad económica impone una nueva realidad social en la que se contrapone el mundo público-productivo frente al mundo privado-reproductivo y relega a los sexos de forma asimétrica a cada uno de estos dominios: el público-productivo para el hombre y el privado-reproductivo para la mujer. Dejando a la mujer bajo la dependencia casi absoluta de los recursos económicos que quedan en las manos de los hombres y responsabilizadas de la crianza de sus descendientes.

En el espacio Público, una conquista relativamente reciente es la incorporación de las mujeres a los estudios universitarios superiores. Haciendo un breve recorrido histórico nos encontramos que en la segunda década del siglo XX tan sólo cien alumnas estudiaban en las universidades españolas (Lameiras, Carrera, Nuñez \& Rodríguez, 2006). Pero serán los últimos 30 años los que marcan de forma decisiva la masiva incorporación de la mujer a los estudios universitarios. Y ya en 1987 las mujeres representaban el 50.34\% de la población universitaria española (INE, 1987-1988). En la actualidad las mujeres representan el 53.67\% del alumnado universitario español, con 807.063 matriculadas, 110.500 más que sus compañeros masculinos (MEC, 2002-2003).

Pero esta presencia de las mujeres en los estudios universitarios no es homogénea, ya que éstas se distribuyen asimétricamente en las diferentes áreas de conocimiento. Los datos disponibles, relativos al curso 2002-2003, muestran que la proporción de mujeres alcanza los porcentajes de $62.29 \%, 66.10 \%$ y 63.8\% en las áreas de Ciencias Sociales y Jurídicas, Ciencias Experimentales y de la Salud, y Humanidades respectivamente. Y finalmente las Enseñanzas Técnicas siguen siendo una asignatura pendiente para las mujeres en las que se encuentran todavía infrarrepresentadas con un porcentaje del 27.30\% (MEC, 2002-2003; Instituto de la Mujer, 2006). Esta situación se reproduce en la mayoría de los países de Europa (ETAN, 2000). Además las mujeres obtienen hoy en día las mejores tasas de rendimiento académico, acaparando el 52.5\% de los premios nacionales de carrera otorgados en el curso 2002-2003 en España ((Lameiras et al., 2006).

Estos datos sobre la masiva presencia de la mujer en los niveles educativos más altos y con buenos resultados académicos, se debería corresponder a nivel laboral. Pero en contra de lo esperado, nos encontramos que las mujeres representan escasamente el 38.7\% de la tasa de ocupación en España en el año 2005, siendo las mujeres inactivas en nuestro país más del doble que los hombres inactivos. De estas mujeres inactivas, el 28,4\% se dedican a las tareas domésticas. Además las mujeres acumulan la mayor tasa de desempleo (en el 2004 el 14,39 \% frente al 7,55 \% de los hombres) y la relación entre menor nivel de estudios y tasa de desempleo es más marcada. Por último, destacar también que las mujeres que consiguen acceder al mundo laboral cobran de media sueldos inferiores a los de sus compañeros con su mismo nivel educativo, con una brecha salarial que ronda el 30\% (Informe Ranstad, 2006).

Por otro lado, la resistencia a la igualdad real entre géneros se constata también en el ámbito privado, ya que la entrada de la mujer en el espacio público no se ha correspondido con el tránsito del hombre al espacio doméstico. En el informe del Instituto de la Mujer Las mujeres en cifras (2001), se realizó un estudio comparativo en función al género de los usos de tiempo dedicados al desarrollo de las tareas domésticas desde 1993 hasta el 2001. Se demuestra que las mujeres dedican mucho más tiempo al trabajo doméstico que los hombres en el 2001 y, ya lo dedicaban en 1993. Las mayores diferencias entre géneros se producen en cuanto al tiempo dedicado al trabajo de la casa como cocinar, planchar, lavar, fregar o quitar el polvo. Las mujeres dedicaban en el 2001 de 3 horas 57 minutos y los hombres 44 minutos; es decir, las mujeres invertían en la ejecución de estas tareas más del quíntuplo de tiempo de los varones. No obstante, es importante destacar que esas diferencias han disminuido desde 1993. En general las mujeres dedicaban 36 minutos menos a las tareas domésticas y los hombres han incrementado en 40 minutos el tiempo que invierten en las tareas domésticas, 
desde el año 1993 hasta el año 2001, destacando este aumento en las tareas propiamente de la casa. Esta tendencia se mantiene en los últimos datos ofrecidos en diversos estudios (Instituto de la Mujer, 2006; Eurostat, 2006) que muestran una disminución en las diferencias de los tiempos que hombres y mujeres dedican a las tareas domésticas aunque las mujeres siguen dedicando a éstas más del doble de tiempo del que dedican los hombres.

Es por tanto el objetivo de este estudio, analizar la división del espacio público y privado del colectivo docente no universitario español, teniendo en cuenta la cobertura del centro educativo (centro de Educación Primaria y centro de Educación Secundaria), la titularidad (centros públicos y centros privados) y el posicionamiento (centros religiosos y centros laicos). Comprobando en el espacio público, la presencia de la mujer y si ostenta cargos de responsabilidad; y en el espacio privado comprobando como los profesores y las profesoras se implican en el desempeño de las tareas domésticas. Nos centramos en analizar las tareas que se llevan a cabo en el hogar por constituir un ámbito fundamental de la convivencia de los géneros y en el que se encuentran las mayores asimetrías.

\section{Método}

\section{Participantes}

En este estudio contamos con una muestra representativa a nivel nacional de 744 docentes no universitarios españoles de los cuales un $62.5 \%$ son profesoras y un $37.4 \%$ son profesores, con un rango de edad que va desde los 22 a los 65 años. La media de edad de este colectivo es de 41.3 (SD: 9.9). El 49.1\% de los docentes pertenecen a la Enseñanza Secundaria (que abarca desde los 12 a los 16 años) y el 50.9\% pertenecen a la Enseñanza Primaria (que va desde los 6 a los 12 años). La aplicación de los cuestionarios se llevó a cabo en 85 centros educativos, de un total de 148 centros contactados, con una tasa de respuesta fue del $57.4 \%$. La selección de la muestra que representa a la población española de docentes, se hizo tomando como variable independiente la tipología del centro, es decir, la cobertura (enseñanza primaria vs. Sexundaria), la titularidad (público vs. privado) y el posicionamiento (religioso vs. laico).

\section{Instrumentos}

Para analizar los espacios público y privado se ha recurrido a los siguientes cuestionarios:

1. Variables sociodemográficas como el género, la edad, la opción religiosa, el nivel de estudios, si asume cargos en el centro y tipo de cargo, si considera necesario una Educación que garantice igualdad, si ha recibido formación sobre coeducación, y si estaría dispuesto a recibir formación sobre coeducación.

2. Tiempo dedicado a las tareas domésticas (adaptado de Martínez, 1995). Este cuestionario está formado por un total de 29 preguntas sobre el tiempo en minutos que se dedica semanalmente a la realización de trabajos domésticos. Para maximizar la utilidad de la escala se realiza un análisis factorial exploratorio de componentes principales con rotación varimax y así conocer cómo se agrupan las diferentes tareas (Medida de adecuación muestral de Kaiser-Meyer-Olkin (KMO)= .894; Prueba de esfericidad de Bartlett: $\mathrm{Chi}^{2}=7704.04$; $\mathrm{p}>$. 001; estos resultados indican que el análisis factorial es adecuado y que el modelo está ajustado). Se obtuvieron los cuatro factores siguientes: a) Tiempo dedicado al hogar: como cocinar, lavar, planchar o coser. b) Tiempo dedicado a los/as hijos/as: como atender a los/as hijos e hijas, o llevarlos a la consulta médica. c) Tiempo dedicado al bricolaje: como pintar, empapelar, o reparar electrodomésticos. d) Tiempo dedicado a tareas puntuales cotidianas: como poner la mesa o sacar la basura.

Se calcula una puntuación total del tiempo dedicado semanalmente a las tareas domésticas y también puntuaciones totales para cada uno de los factores resultantes. 


\section{Resultados}

Perfil del docente no universitario en función del género

Debido a la importancia de la variable género en el tema que nos concierne, se estudia para cada variable las diferencias significativas. El 62.5\% del colectivo docente son mujeres y el 37.4\% hombres (ver tabla 1). En lo referente a la opción religiosa (ver tabla 1), destacar que el 42.5\% del colectivo docente se declaró católico no practicante; un importante porcentaje se declaró católico practicante (38,9\%); y el 18,4\% no creyente. En función al género se han encontraron diferencias significativas $\left(\mathrm{Chi}^{2}=8.70 ; \mathrm{p}<.01\right)$, en cuanto que son las profesoras las que se declaran católicas practicantes (42.2\%) en mayor medida que sus compañeros (36.2\%).

El 86.6\% de los/as docentes son licenciados y 13.4\% diplomados, pero al comparar el nivel de estudios en función del género encontramos diferencias significativas $\left(\mathrm{Chi}^{2}=9.95 ; \mathrm{p}<.001\right)$, ya que el $62.1 \%$ de las profesoras son diplomadas y el $50.2 \%$ de los profesores son licenciados (ver tabla 1).

Del total de entrevistados/as, el 24,5\% ostenta un cargo en el centro. Al estudiar el perfil de las personas que ocupan los cargos en los diferentes centros se observó que, a pesar de ser una población donde se da una mayor presencia de mujeres, hay más hombres $(\mathrm{n}=90)$ que ocupen cargos que las mujeres ( $\mathrm{n}=84$ ). Esto significa que el 33.1\% de los profesores ocupan cargos en el centro frente al $18.6 \%$ de sus compañeras. Diferencias que son significativas $\left(\mathrm{Chi}^{2}=19.56\right.$; $\left.\mathrm{p}<.001\right)$ y analizamos el tipo de cargo que ocupan, nos encontramos que el puesto de Director, el de mayor rango, lo ocupan en un $19.5 \%$ de los profesores frente al 6.8\% las profesoras $\left(\mathrm{Chi}^{2}=6.72 ; \mathrm{p}<.05\right)$.

Centrándonos en el tema de la coeducación (ver tabla 1), son las profesoras las que ven la coeducación como una necesidad importante para garantizar la igualdad entre géneros $(t=2.29 ; p<.05)$. Al mismo tiempo, son las profesoras las que más formación han recibido sobre la coeducación, y son además las que están más dispuestas a recibir y a seguir recibiendo formación en relación a la igualdad entre hombres y mujeres $\left(\mathrm{Chi}^{2}=3.90 ; \mathrm{p}<.01\right)$.

Tabla 1. Perfil sociodemográfico de docentes no universitarios en función al género, la cobertura, la titularidad y el posicionamiento del centro educativo

\begin{tabular}{|c|c|c|c|c|}
\hline & & & & \\
\hline & Mujer & Hombre & Secundaria & Primaria \\
\hline Género & & & & \\
\hline Mujer & 6250 & & $44.3 \%$ & $55.7 \%$ \\
\hline Hombre & 0..5\% & $37.4 \%$ & $57.8 \%$ & $42.2 \%$ \\
\hline & & & & \\
\hline Edad (Media) & 40.9 & 41.8 & 40.4 & 42 \\
\hline & & & & \\
\hline Opción Religiosa & & & & \\
\hline Practicante & $42.2 \%$ & $36.2 \%$ & $31.8 \%$ & $47.7 \%$ \\
\hline No practicante & $43.7 \%$ & $41.2 \%$ & $45.1 \%$ & $40.2 \%$ \\
\hline No creyente & $14.1 \%$ & $22.7 \%$ & $23.1 \%$ & $12.1 \%$ \\
\hline & & & & \\
\hline $\begin{array}{l}\text { Nivel de estudios } \\
\text { Diplomado/a }\end{array}$ & $50.2 \%$ & $37.9 \%$ & $14.5 \%$ & $73.7 \%$ \\
\hline Licenciado/a & $49.8 \%$ & ** $62.1 \%$ & $85.5 \%$ & * $26.3 \%$ \\
\hline Cargo en Centro & & & & \\
\hline No & $81.4 \%$ & $66.9 \%$ & $67.8 \%$ & $83.8 \%$ \\
\hline $\mathrm{Si}$ & $18.6 \%$ & ** $33.1 \%$ & $32.2 \%$ & * $16.2 \%$ \\
\hline
\end{tabular}




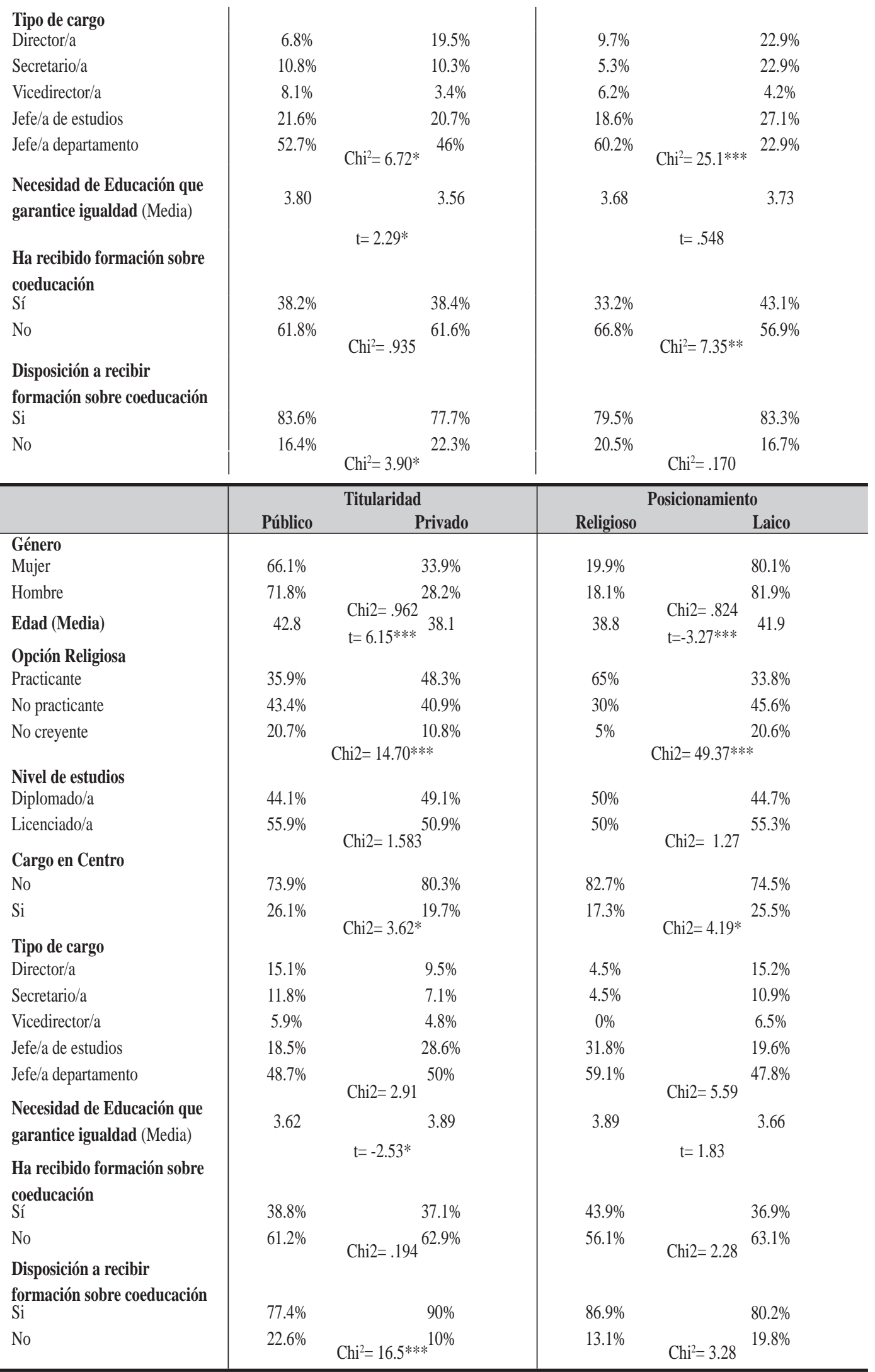

Nota: ${ }^{*} \mathrm{p}<.05 ;{ }^{* *} \mathrm{p}<.01 ; * * * \mathrm{p}<.001$ 
En relación a la Cobertura del centro (ver tabla 1), de Enseñanza Secundaria y Primaria, encontramos que hay un mayor porcentaje de hombres (57.8\%) en Secundaria y de mujeres (55.7\%) en Primaria $\left(\mathrm{Chi}^{2}=90.4 ; \mathrm{p}<.05\right)$. En cuanto a la opción religiosa, el 23.1\% de los/as docentes de Enseñanza Secundaria se declaran no creyentes frente al $47.7 \%$ de los/as docentes de Primaria que se confiesan católicos practicantes $\left(\mathrm{Chi}^{2}=24.4 ; \mathrm{p}<.001\right)$. La titulación de los/as docentes de Secundaría (85.5\%) es mayoritariamente de licenciados, frente a los/as docentes de Primaria (73.7\%) que son diplomados $\left(\mathrm{Chi}^{2}=12.1 ; \mathrm{p}<.05\right)$.

Son los/as docentes de Secundaria (32.2\%) los que más cargos ostentan frente a los de Primaria (16.2\%), $\left(\mathrm{Chi}^{2}=25.6 ; \mathrm{p}<.001\right)$, aunque la mayoría de los/as docentes de Secundaria son Jefes/as de Departamentos (60.2\%) y el 22.9\% de los/as docentes de Primaria son Directores/as, $\left(\mathrm{Chi}^{2}=25.1\right.$; $\mathrm{p}<.001)$. Fueron los/as docentes de Secundaria (66.8\%) los que han recibido menos formación en coeducación que los de Primaria (56.9\%), $\left(\mathrm{Chi}^{2}=7.35 ; \mathrm{p}<.01\right)$, pero en general, tanto los/as docentes de Primaria como los de Secundaria estarían interesados en recibir formación en relación a la igualdad entre hombres y mujeres.

La segunda taxonomía del centro hace referencia a la Titularidad (Enseñanza Pública y Privada). Son los/as docentes de la enseñanza privada más jóvenes, con una media de 38.1 años, que los de la enseñanza pública (42.8 años), ( $\mathrm{t}=6.15 ; \mathrm{p}<.001)$. Esto podría venir explicado por el acceso a la plaza de enseñanza Pública, por concurso-oposición. En cuanto a la opción religiosa, son los/as docentes de la enseñanza Privada los que en un $48.3 \%$ se confiesan católicos practicantes, frente a un $35.9 \%$ de docentes de la enseñanza pública $\left(\mathrm{Chi}^{2}=14.7 ; \mathrm{p}<.001\right)$. Esto se debe a que un gran número de los Centros Privados son de carácter religioso. Los/as docentes de centros Públicos (26.1\%) ocupan más cargos que los/as docentes de colegios Privados (19.7\%), $\left(\mathrm{Chi}^{2}=3.62 ; \mathrm{p}<.05\right)$.

En relación a la coeducación, son los/as docentes de colegios Privados los que consideran más importante llevar a cabo una educación que garantice la igualdad entre géneros $(\mathrm{t}=-2.53 ; \mathrm{p}<.05)$; al mismo tiempo, que están más dispuestos/as a recibir formación sobre coeducación (90\%) frente a los/as docentes de centros Públicos (77.4\%), $\left(\mathrm{Chi}^{2}=16.5\right.$; p<.001). Destacar también, que los/as docentes de centros Públicos (64\%) tienen más hijos/as que los de centros Privados (49.6\%), (Chi ${ }^{2}=$ 13.6; $\mathrm{p}<.001$ ); este resultado se podría explicar por las diferencias en la edad entre los/as docentes de centros públicos y privados, ya que estos últimos son más jóvenes.

Por último, la tercera tipología del centro hace referencia al Posicionamiento (Religioso y Laico). Son los/as docentes pertenecientes a centros Religiosos, más jóvenes (38.8 años) que los de centros Laicos (41.9 años), ( $\mathrm{t}=-3.27 ; \mathrm{p}<.001)$. A su vez, los/as docentes de centros Religiosos se manifestaron más religiosos en un 65\%, $\left(\mathrm{Chi}^{2}=49.3\right.$; $\left.\mathrm{p}<.001\right)$.

En relación a su situación laboral, un 25.5\% de los/as docentes de enseñanza Laica ocupan algún cargo en el centro frente al 17.3\% de los/as docentes de enseñanza Religiosa $\left(\mathrm{Chi}^{2}=4.19 ; \mathrm{p}<.05\right)$.

Tiempo que dedican los y las docentes a las tareas domésticas

En relación a los tiempos dedicados semanalmente a las Tareas Domésticas, las mujeres, en este caso las profesoras, son las que sobrellevan más cargas en la ejecución de este tipo de tareas, dedicando un total de 1344.5 minutos en total, que equivalen a 22.4 horas semanales y a 3.2 horas al día. Por su parte los profesores dedican un total de 1054 minutos, que equivalen a 17.65 horas semanales y a 2.5 horas al día. A continuación, analizamos estos datos de forma más detallada en función a las cuatro subescalas resultantes del análisis factorial: Tiempo dedicado a Tareas de Hogar, a Hijos/as, al Bricolaje y a Tareas Puntuales.

En cuanto al tiempo dedicado a las Tareas del Hogar, la media de tiempo invertido es de 678 minutos semanalmente. Claramente son las mujeres las que más tiempo dedican a estás tareas, 829 minutos frente a 449 minutos de los hombre $(\mathrm{t}=7.91 ; \mathrm{p}<.001)$ (ver tabla 2$)$. 
Tabla 2. Diferencias de medias (desviación típica) de las escalas de Tiempo dedicado semanalmente a Tareas Domésticas en función del género, la Cobertura, la Titularidad y el Posicionamiento del Centro Educativo

\begin{tabular}{|c|c|c|c|c|c|c|c|c|}
\hline & \multicolumn{2}{|c|}{ Género } & \multicolumn{2}{|c|}{ Titularidad } & \multicolumn{2}{|c|}{ Posicionamiento } & \multicolumn{2}{|c|}{ Cobertura } \\
\hline & Mujer & Hombre & Público & Privado & Laico & Religioso & Secundaria & Primaria \\
\hline $\begin{array}{l}\text { Tiempo dedicado } \\
\text { a tareas hogar }\end{array}$ & $\begin{array}{c}829 \\
(731)\end{array}$ & $\begin{array}{c}449 \\
(416)\end{array}$ & $\begin{array}{c}703 \\
(695)\end{array}$ & $\begin{array}{c}641 \\
(566)\end{array}$ & $\begin{array}{c}698 \\
(678)\end{array}$ & $\begin{array}{c}623 \\
(562)\end{array}$ & $\begin{array}{c}614 \\
(512)\end{array}$ & $\begin{array}{c}750 \\
(766)\end{array}$ \\
\hline \multirow[t]{2}{*}{$\begin{array}{l}\text { Tiempo dedicado } \\
\text { a hijos/as }\end{array}$} & $\begin{array}{l}t=7 \\
307 \\
(612)\end{array}$ & $\begin{array}{l}\text { *** } \\
284 \\
(512)\end{array}$ & $\begin{array}{l}279 \\
(549)\end{array}$ & $\begin{array}{c}18 \\
333 \\
(628)\end{array}$ & $\begin{array}{c}288 \\
(559)\end{array}$ & $\begin{array}{r}.22 \\
333 \\
(642)\end{array}$ & $\begin{array}{l}350 \\
(603)\end{array}$ & $\begin{array}{c}246 \\
(544)\end{array}$ \\
\hline & \multicolumn{2}{|c|}{$\mathrm{t}=.510$} & \multicolumn{2}{|c|}{$t=-1.21$} & \multicolumn{2}{|c|}{$\mathrm{t}=.844$} & \multicolumn{2}{|c|}{$\mathrm{t}=.844$} \\
\hline \multirow[t]{2}{*}{$\begin{array}{l}\text { Tiempo dedicado } \\
\text { a bricolaje }\end{array}$} & $\begin{array}{c}131 \\
(219)\end{array}$ & $\begin{array}{c}205 \\
(257)\end{array}$ & $\begin{array}{l}165 \\
(250)\end{array}$ & $\begin{array}{c}142 \\
(202)\end{array}$ & $\begin{array}{c}162 \\
(244)\end{array}$ & $\begin{array}{c}141 \\
(197)\end{array}$ & $\begin{array}{l}165 \\
(244)\end{array}$ & $\begin{array}{l}151 \\
(228)\end{array}$ \\
\hline & \multicolumn{2}{|c|}{$\mathrm{t}=-4.16^{* * *}$} & \multicolumn{2}{|c|}{$t=-1.26$} & \multicolumn{2}{|c|}{$\mathrm{t}=.959$} & \multicolumn{2}{|c|}{$t=-.959$} \\
\hline $\begin{array}{l}\text { Tiempo } \\
\text { dedicado a } \\
\text { tareas puntuales }\end{array}$ & $\begin{array}{r}77.5 \\
(133) \\
t=-\end{array}$ & $\begin{array}{c}121 \\
(122) \\
0 * * *\end{array}$ & $\begin{array}{l}97.7 \\
(136) \\
t=\end{array}$ & $\begin{array}{r}85.2 \\
(118) \\
1\end{array}$ & $\begin{array}{r}94.3 \\
(133) \\
t\end{array}$ & $\begin{array}{r}91.7 \\
(118) \\
211\end{array}$ & $\begin{array}{r}94.08 \\
(113) \\
t=-.\end{array}$ & $\begin{array}{l}93.53 \\
(146)\end{array}$ \\
\hline
\end{tabular}

Nota: ${ }^{*} \mathrm{p}<.05 ; * * \mathrm{p}<.01 ; * * * \mathrm{p}<.001$

Si analizamos los tiempos dedicados semanalmente a las Tareas del Hogar en cada tipología de centro en función del género (ver tabla 3), no encontramos diferencias en los tiempos, ya que siguen siendo las profesoras de Enseñanza Primaria $(\mathrm{t}=7.20 ; \mathrm{p}<.001)$, de Enseñanza Secundaria $(\mathrm{t}=3.33$; $\mathrm{p}<.001)$, de Centros Públicos ( $\mathrm{t}=6.69 ; \mathrm{p}<.001)$, de centros Privados $(\mathrm{t}=4.41 ; \mathrm{p}<.001)$, de Centros Religiosos ( $\mathrm{t}=3.63 ; \mathrm{p}<.001)$ y de centros Laicos $(\mathrm{t}=7.06$; $\mathrm{p}<.001)$, las que dedican más tiempo al desarrollo de las Tareas de Hogar que sus compañeros.

En cuanto al tiempo dedicado a los/as Hijos/as, la media del tiempo invertido por los/as docentes es de 297 minutos semanalmente. En principio no se han detectado diferencias significativas en los tiempos de los profesores y las profesoras (ver tabla 2). Sin embargo en función a la Cobertura del centro, son los/as docentes de Secundaria los que dedican más tiempo al cuidado y atención de sus hijos/as que los de Primaria ( $\mathrm{t}=-2.46 ; \mathrm{p}<.05$ ), esto puede deberse a la mayor flexibilidad del horario laboral de los/as docentes de Secundaria. Por otro lado, las profesoras de la Enseñanza Primaria dedican más tiempo a sus hijos/as que sus compañeros ( $\mathrm{t}=2.77 ; \mathrm{p}<.01)$ (ver tabla 3 ).

Tabla 3. Diferencias de medias (desviación típica) en función del género de las escalas del Tiempo dedicado semanalmente a Tareas Domésticas en relación a la Cobertura, el Posicionamiento y la Titularidad del Centro

\begin{tabular}{|c|c|c|c|c|c|c|c|c|c|c|c|c|}
\hline & \multicolumn{4}{|c|}{ Cobertura } & \multicolumn{4}{|c|}{ Posicionamiento } & \multicolumn{4}{|c|}{ Titularidad } \\
\hline & \multicolumn{2}{|c|}{ Secundaria } & \multicolumn{2}{|c|}{ Primaria } & \multicolumn{2}{|c|}{ Religioso } & \multicolumn{2}{|c|}{ Laico } & \multicolumn{2}{|c|}{ Público } & \multicolumn{2}{|c|}{ Privado } \\
\hline & M & $\mathrm{H}$ & M & $\mathrm{H}$ & M & $\mathrm{H}$ & M & $\mathbf{H}$ & M & $\mathrm{H}$ & M & $\mathrm{H}$ \\
\hline \multirow[t]{2}{*}{$\begin{array}{l}\text { Tiempo dedicado } \\
\text { al Hogar }\end{array}$} & $\begin{array}{c}692 \\
(563)\end{array}$ & $\begin{array}{c}514 \\
(419)\end{array}$ & $\begin{array}{c}937 \\
(826)\end{array}$ & $\begin{array}{c}359 \\
(396)\end{array}$ & $\begin{array}{c}749 \\
(611)\end{array}$ & $\begin{array}{c}405 \\
(371)\end{array}$ & $\begin{array}{c}848 \\
(758)\end{array}$ & $\begin{array}{c}459 \\
(426)\end{array}$ & $\begin{array}{c}865 \\
(797)\end{array}$ & $\begin{array}{c}458 \\
(393)\end{array}$ & $\begin{array}{c}757 \\
(578)\end{array}$ & $\begin{array}{c}423 \\
(472)\end{array}$ \\
\hline & \multicolumn{2}{|c|}{$\mathrm{t}=3.33^{* * *}$} & \multicolumn{2}{|c|}{$\mathrm{t}=7.20 * * *$} & \multicolumn{2}{|c|}{$\mathrm{t}=3.63^{* * *}$} & \multicolumn{2}{|c|}{$\mathrm{t}=7.06^{* * *}$} & \multicolumn{2}{|c|}{$\mathrm{t}=6.69 * * *$} & \multicolumn{2}{|c|}{$\mathrm{t}=4.41 * * *$} \\
\hline \multirow[t]{2}{*}{$\begin{array}{l}\text { Tiempo dedicado } \\
\text { hijos/as }\end{array}$} & $\begin{array}{c}314 \\
(598)\end{array}$ & $\begin{array}{c}395 \\
(607)\end{array}$ & $\begin{array}{c}301 \\
(624)\end{array}$ & $\begin{array}{c}133 \\
(781)\end{array}$ & $\begin{array}{c}311 \\
(659)\end{array}$ & $\begin{array}{c}381 \\
(620)\end{array}$ & $\begin{array}{c}306 \\
(601)\end{array}$ & $\begin{array}{c}263 \\
(485)\end{array}$ & $\begin{array}{c}286 \\
(589)\end{array}$ & $\begin{array}{c}270 \\
(485)\end{array}$ & $\begin{array}{c}346 \\
(656)\end{array}$ & $\begin{array}{c}319 \\
(576)\end{array}$ \\
\hline & \multicolumn{2}{|c|}{$t=-1.27$} & \multicolumn{2}{|c|}{$\mathrm{t}=2.77 * *$} & \multicolumn{2}{|c|}{$t=-.618$} & \multicolumn{2}{|c|}{$t=.902$} & \multicolumn{2}{|c|}{$t=.320$} & \multicolumn{2}{|c|}{$t=.302$} \\
\hline
\end{tabular}




\begin{tabular}{|c|c|c|c|c|c|c|c|c|c|c|c|c|}
\hline \multirow[t]{2}{*}{$\begin{array}{l}\text { Tiempo } \\
\text { dedicado } \\
\text { Bricolaje }\end{array}$} & $\begin{array}{c}129 \\
(214)\end{array}$ & $\begin{array}{c}211 \\
(272)\end{array}$ & $\begin{array}{c}133 \\
(223)\end{array}$ & $\begin{array}{c}196 \\
(236)\end{array}$ & $\begin{array}{c}113 \\
(156)\end{array}$ & $\begin{array}{c}196 \\
(249)\end{array}$ & $\begin{array}{c}135 \\
(232)\end{array}$ & $\begin{array}{c}207 \\
(260)\end{array}$ & $\begin{array}{c}134 \\
(239)\end{array}$ & $\begin{array}{c}215 \\
(261)\end{array}$ & $\begin{array}{c}125 \\
(174)\end{array}$ & $\begin{array}{c}179 \\
(247)\end{array}$ \\
\hline & \multicolumn{2}{|c|}{$\mathrm{t}=3.22 * * *$} & \multicolumn{2}{|c|}{$t=-2.50 *$} & \multicolumn{2}{|c|}{$\mathrm{t}=-2.42 *$} & \multicolumn{2}{|c|}{$\mathrm{t}=-3.50^{* * *}$} & \multicolumn{2}{|c|}{$\mathrm{t}=-3.58 * * *$} & \multicolumn{2}{|c|}{$t=-1.91$} \\
\hline \multirow[t]{2}{*}{$\begin{array}{l}\text { Tiempo } \\
\text { dedicado a } \\
\text { tareas puntuales }\end{array}$} & $\begin{array}{c}68 \\
(103)\end{array}$ & $\begin{array}{c}126 \\
(117)\end{array}$ & $\begin{array}{c}84.6 \\
(152)\end{array}$ & $\begin{array}{c}115 \\
(130)\end{array}$ & $\begin{array}{c}83 \\
(125)\end{array}$ & $\begin{array}{c}108 \\
(105)\end{array}$ & $\begin{array}{c}75 \\
(135)\end{array}$ & $\begin{array}{c}125 \\
(126)\end{array}$ & $\begin{array}{c}76 \\
(139)\end{array}$ & $\begin{array}{c}131 \\
(126)\end{array}$ & $\begin{array}{c}79 \\
(121)\end{array}$ & $\begin{array}{c}98 \\
(111)\end{array}$ \\
\hline & \multicolumn{2}{|c|}{$\mathrm{t}=-5.01 * * *$} & \multicolumn{2}{|c|}{$\mathrm{t}=-1.99 *$} & \multicolumn{2}{|c|}{$t=-1.16$} & \multicolumn{2}{|c|}{$\mathrm{t}=-4.41 * * *$} & \multicolumn{2}{|c|}{$\mathrm{t}=-4.48 * * *$} & \multicolumn{2}{|c|}{$t=-1.12$} \\
\hline
\end{tabular}

Nota: ${ }^{*} p<.05 ;{ }^{* \star} p<.01 ;{ }^{* \star *} \mathrm{p}<.001 ; \mathrm{M}$ : mujer y $\mathrm{H}$ : hombre.

El tiempo dedicado al Bricolaje fue de una media de 158 minutos semanales y parece ser una tarea ejecutada en mayor medida por los hombres (ver tabla 2). En cuanto que son ellos los que doblan el tiempo dedicado por la mujeres a la realización de tareas de bricolaje $(t=-4.16 ; \mathrm{p}<.001)$. Este dato se confirma al comparar profesores y profesoras en función de la tipología del centro (ver tabla 3). De forma que, son los profesores de la Enseñanza Primaria ( $t=-2.50 ; \mathrm{p}<.05)$, de Enseñanza Secundaria $(\mathrm{t}=3.22 ; \mathrm{p}<.001)$, de Centros Públicos $(\mathrm{t}=-3.58 ; \mathrm{p}<.001)$, de Centros Religiosos $(\mathrm{t}=$ $-2.42 ; \mathrm{p}<.05)$ y de Centros Laicos $(\mathrm{t}=-3.50 ; \mathrm{p}<.001)$, los que dedican más tiempo al desempeño de tareas relacionadas al bricolaje como pintar, empapelar, hacer pequeños arreglos de albañilería y electricidad, decorar la casa o reparar electrodomésticos entre otras.

Las tareas puntuales como sacar la basura o poner la mesa fueron tareas realizadas principalmente por profesores, que dedican semanalmente un total de 121 minutos frente a los 77.5 minutos de las profesoras $(\mathrm{t}=-4.50 ; \mathrm{p}<.001)$ (ver tabla 2). Al comparar profesores y profesoras en función de la tipología del centro (ver tabla 5.51), se confirma el resultado anterior. De forma que, son los profesores de la Enseñanza Primaria ( $t=-1.99 ; \mathrm{p}<.05)$, de Enseñanza Secundaria $(t=-5.01 ; \mathrm{p}<.001)$, de Centros Públicos ( $\mathrm{t}=-4.48 ; \mathrm{p}<.001)$ y de Centros Laicos $(\mathrm{t}=-4.41 ; \mathrm{p}<.001)$ los que más tiempo pasan realizando este tipo de tareas cotidianas (ver tabla 3 ).

\section{Discusión}

El ámbito educativo es uno de los ámbitos laborales que tiene una mayor presencia femenina, representando éstas el $70 \%$ del total de docentes no universitarios; superando incluso el 75\% de presencia femenina tanto en la Educación Infantil como en la Educación Primaria (Instituto de la Mujer, 2006). Partiendo de este hecho, se justifica que la muestra de docentes se encuentre sobrerrepresentada por mujeres, con un $62.5 \%$ de profesoras. Sin embargo a la hora de identificar el nivel de estudios se comprueba que la mayoría de las profesoras son diplomadas mientras que sus compañeros son licenciados, y además son ellos los que siguen asumiendo los puestos de mayor poder en el centro educativo como el de Director.

En relación a la tipología del centro se constata que los y las docentes que pertenecen a la Enseñanza Secundaria ocupan más puestos en el centro educativo que los de Educación Primaria. Pero siguen siendo los hombres, los que acaparan más cargos en los Centros Educativos, tanto de Educación Primaria como de Educación Secundaria. En general, los y las docentes pertenecientes a Centros Religiosos desempeñan pocos cargos, debido a que en muchos Centros Religiosos los cargos, como Director o Vicedirector, están ocupados por miembros de la congregación religiosa a la que está adscrita el Centro Educativo.

En este sentido, nuestros resultados coinciden con el estudio de Callejo et al. (2004) señala que las cifras reflejan la asimetría existente entre los y las docentes sobre la distribución de cargos, a pesar de que la norma de igualdad entre los géneros aparece como algo indiscutible en el sistema educativo. Lo sorprendente es que nadie lo cuestiona y así el sistema educativo no lo percibe como un problema propio sino más bien como un problema externo que repercute en él. De modo que el acceso formal de las profesoras a cargos directivos no se percibe obstaculizado, sino que es la trayectoria profesional en sí misma y la interpenetración entre la representación colectiva y la 
práctica del poder y la representación colectiva y la práctica de la diferencia entre los géneros, la que explicaría la ausencia de mujeres en cargos directivos. Pero si su mejor predisposición a ocupar cargos de responsabilidad que tengan un carácter más subordinado, y que explicaría que las profesoras prefieran ser jefas de estudio que directoras. Las conclusiones de este estudio plantean que la mujer en este ámbito educativo no universitario no tiene limitaciones de acceso, sino que parece que se autoexcluye o más bien que no le interesa ocuparlo. Ya que un cargo de directivo implica además de mayor responsabilidad, una mayor disponibilidad temporal, lo cual conlleva que la mujer debe invertir más tiempo a su trabajo y en cierta forma, restarlo del tiempo dedicado a su familia (Callejo et al., 2004). Este hecho es el que "impide" que las mujeres decidan ocupan cargos en el centro, factores familiares que no parecen interferir en la proyección a cargos de sus compañeros.

En relación a la opción religiosa muchos de los y las docentes se declaran católicos no practicantes, pero siguen siendo las mujeres más religiosas que los hombres independientemente de la Cobertura, la Titularidad y el Posicionamiento del centro, tendencia que apuntan estudios previos (Sidanius, 1993; Harville y Rienzi, 2000; Glick, Lameiras y Rodríguez, 2002). Aunque los y las docentes más religiosos son los que pertenecen a centros Privados y Religiosos.

Tal y como señalan Callejo et al. (2004) el sistema educativo se convierte en un refugio laboral privilegiado para las mujeres ya que sus condiciones de horario y de disponibilidad de tiempo les permite desarrollar una profesión y al mismo tiempo ocuparse de sus hijos/as y de las tareas domésticas. Este dato también lo confirmamos en nuestro estudio, las profesoras son las que sobrellevan más carga: dedican 3.2 horas al día a las tareas domésticas frente a los profesores que dedican una media de 2.5 horas al día. De forma más específica podemos destacar: que las profesoras doblan el tiempo dedicado a tareas del Hogar a sus compañeros; que profesores y profesoras dedican el mismo tiempo a la atención y cuidado de sus hijos/as; y, que son los profesores los que pasan más tiempo dedicado a tareas de bricolaje y tareas puntuales. Los estudios siguen constatando que la mujer invierte más tiempo a las tareas domésticas que los hombres (Lennon y Rosenfield, 1994; Lafuente, Barberá y Sarrio, 1998; Instituto de la Mujer, 2001, 2006; Informe Eurostat, 2006).

En relación al tema de la coeducación los resultados confirman que no interesa por igual a profesores y a profesoras. En general las profesoras ven más necesaria la formación en coeducación, y están más dispuestas a recibir formación específica del tema, para poder garantizar una educación de igualdad entre hombres y mujeres. De esta forma, son las profesoras de Primaria frente a las de Secundaria, las que están más interesadas en los temas relativos a la coeducación. Mientras que a los profesores y a las profesoras de centros religiosos y privados el tema de la coeducación no parece preocuparles. Esto pone de manifiesto que la ideología de un centro se relaciona con las actitudes hacia los temas relacionados con la igualdad entre géneros. De forma que estudios como el de Glick et al. (2002) así lo manifiestan.

Por consiguiente, en este estudio en relación al análisis del perfil docente se manifiesta claramente la dicotomía público versus privado. Sorprende que aunque la enseñanza es una de las ramas consideradas tradicionalmente femeninas, los profesores, independientemente de la Cobertura, la Titularidad o el Posicionamiento del Centro, son los que siguen ostentando los cargos directivos, es decir, siguen acaparando los puestos de poder dentro del Sistema Educativo. Frente a las profesoras, que aunque son las que están más satisfechas con su trabajo también son las siguen dedicando más tiempo a la ejecución de las tareas domésticas. Lo que demuestra que la incorporación de la mujer a la esfera pública no va asociada a una mayor implicación de los hombres en la esfera privada, en este caso dentro del ámbito educativo. 


\section{Referencias}

Callejo, G. J., Gómez, E. C., \& Casado, A. E. (2004). El techo de cristal en el sistema educativo. Madrid: UNED.

Glick, P., Lameiras, M., \& Rodríguez, Y. (2002). Education and religiosity as predictors of ambivalently sexist attitudes. Sex Roles, 47 (9/10), 433-441

Harville, M. L., \& Rienzi, B. (2000). Equal worth and gracious submission: Judeo-Christian acttitudes toward employed women. Psychology of Women Quarterly, 24, 145-147.

I.N.E. (1987-1988). Instituto Nacional de Estadística. Estadística de la Enseñanza Superior en España. Madrid: INE.

Informe ETAN (European Technology Assessment Network) (2000). Science Policies in the European Union: Promotioning Excellence through Mainstreaming Gender Equality. Luxemburgo: OPOCE.

Informe Eurostat (2006). How is the time of women and men distributed in Europe? Statistics in focus - Population and social conditions. 4/2006.

Informe Randstad (2006). VI Informe Calidad del trabajo en la Europa de los quince. Mujer y Trabajo. Instituto de Estudios Laborales: ESADE.

Instituto de la Mujer (2001). La mujer en cifras (1996-2001). Madrid: Instituto de la Mujer, MTAS.

Instituto de la Mujer (2006). Informe hombres y mujeres en España 2006. Madrid: Instituto de la Mujer, MTAS.

Lafuente, M. J., Barberá, E., \& Sarrio, M. (1998). Mujeres, éxito laboral y apoyo familiar. Valencia: Promolibro.

Lameiras, M., Carrera, V., Nuñez, A. \& Rodríguez, Y. (2006). Evolución de la excelencia universitaria demostrada por las mujeres españolas el período 1985-2003. Cuadernos de Género e Tecnología, 1, 29-42.

M.E.C. (2002-2003). Ministerio de Educación y Ciencia. Estadística de la Enseñanza en España. Madrid: MEC.

Martínez, M. C. (1995). Análisis los usos del tiempo dedicado a las tareas domésaticas. Madrid: Instituto de la Mujer.

Sidanius, J. (1993). The interface between racism and sexism. Journal of Psychology, 127, 211322. 